\title{
Bounds on the tensor rank
}

\author{
Edoardo Ballico ${ }^{1}$. Alessandra Bernardi ${ }^{1}$ (D) \\ Luca Chiantini $^{2}$. Elena Guardo ${ }^{3}$
}

Received: 5 May 2017 / Accepted: 16 April 2018 / Published online: 24 April 2018

(C) Fondazione Annali di Matematica Pura ed Applicata and Springer-Verlag GmbH Germany, part of Springer Nature 2018

\begin{abstract}
We give a sufficient criterion for a lower bound of the cactus rank of a tensor. Then we refine that criterion in order to be able to give an explicit sufficient condition for a non-redundant decomposition of a tensor to be minimal and unique.
\end{abstract}

Keywords Tensor rank · Identifiability $\cdot$ Comon conjecture

Mathematics Subject Classification 14 N05 $\cdot$ 15A72

\section{Introduction}

The study of minimal decompositions of a given tensor $T$ as a linear combination of rank one tensors is a hot topic in many areas, ranging from pure algebraic geometry to applications to signal processing, big data analysis, quantum information, etc.

\footnotetext{
Alessandra Bernardi alessandra.bernardi@unitn.it

Edoardo Ballico

edoardo.ballico@unitn.it

Luca Chiantini

luca.chiantini@unisi.it

Elena Guardo

guardo@dmi.unict.it

1 Dipartimento di Matematica, Università di Trento, Trento, Italy

2 Dipartimento di Ingegneria dell'Informazione e Scienze Matematiche, Università di Siena, Siena, Italy

3 Dipartimento di Matematica e Informatica, Università di Catania, Catania, Italy
} 
Vectors $v_{j, i} \in \mathbb{C}^{n_{j}+1}, j=1, \ldots, k, i=1, \ldots, r$, such that

$$
T=\sum_{i=1}^{r} \lambda_{i} v_{1, i} \otimes \cdots \otimes v_{k, i}
$$

for some $\lambda_{i} \in \mathbb{C} \backslash\{0\}$ determine a decomposition of $T$. We will say that the decomposition is non-redundant (cf. Definition 2.1) if we cannot extract any proper subset of $\left\{v_{1, i} \otimes \cdots \otimes\right.$ $\left.v_{k, i}\right\}_{i=1, \ldots, r}$ which generates $T$.

Since we will use geometric arguments through the paper, we use a geometric notation. Thus we identify (up to scalar multiplication) a tensor $T$ with a point in the projective space $\mathbb{P}\left(\mathbb{C}^{n_{1}+1} \otimes \cdots \otimes \mathbb{C}^{n_{k}+1}\right)$ and a decomposition of $T$ with a finite subset $S$ of the Segre embedding of the abstract product $\mathbb{P}\left(\mathbb{C}^{n_{1}+1}\right) \times \cdots \times \mathbb{P}\left(\mathbb{C}^{n_{k}+1}\right)=\mathbb{P}^{n_{1}} \times \cdots \times \mathbb{P}^{n_{k}}$, such that $T$ belongs to the linear span of $S$.

Clearly, having a non-redundant decomposition of a given tensor $T$ does not imply that such a decomposition is minimal, i.e., it has a minimal number of addenda (so that $r$ is the $\operatorname{rank} \operatorname{rk}(T)$ of $T$, cf. Definition 2.3).

In this paper, we give a criterion that certifies if a non-redundant decomposition of a general tensor $T$ is also minimal, thus that it computes the rank of $T$ (cf. Theorem 3.1). Moreover, under certain conditions, we can also show that a decomposition is the unique minimal decomposition of $T$ (cf. Theorem 4.6).

These two facts rely on our main result Theorem 3.1, where we give a criterion to find a lower bound for the cactus rank (cf. 2.3). The idea is geometrically quite simple: assume one has a non-redundant decomposition $S$ of a tensor $T \in \mathbb{P}\left(\mathbb{C}^{n_{1}+1} \otimes \cdots \otimes \mathbb{C}^{n_{k}+1}\right)$, then one can flatten the product $\mathbb{P}^{n_{1}} \times \cdots \times \mathbb{P}^{n_{k}}$ in a partition of two factors and study the geometry of the two projections of $S$, to get the result.

One can easily compare our result with the Kruskal's result on the identifiability of tensors ([15]), which implies a criterion for the minimality of a decomposition. It turns out that our criterion is geometrically simpler, and it applies in a wider range of numerical cases.

Certainly the idea of considering projections of tensors to factors of some partition of the Segre product is not new. What we would like to propose here is a systematic analysis of the geometry of these projections (e.g., an analysis of the Segre function, see Definition 4.1) which, as we demonstrate with our results, can determine new information on deep properties of tensors.

Theorem 3.1 has the consequence that if a non-redundant decomposition projects onto two linearly independent subset in the flattening, then it is also a minimal one (cf. Example 3.6). This can be considered as a step toward the celebrated Strassen's conjecture on the rank of a block tensor (cf. [19]).

Although we are well aware of the recent announcement of a counterexample ([17]) on the so-called Comon's conjecture (the equality between the rank and the symmetric rank of a symmetric tensor $T$ ), we like to emphasize that our Theorem 3.1 provides new examples where such a conjecture holds. In a wide numerical range, if we know the existence of a symmetric decomposition of $T$ with sufficiently general geometric properties, then the conjecture holds for $T$ (cf. Corollary 3.10). For instance, the Corollary applies for general tensors in $\mathbb{P}\left(\operatorname{Sym}^{6}\left(\mathbb{C}^{3}\right)\right)$ and $\mathbb{P}\left(\operatorname{Sym}^{8}\left(\mathbb{C}^{3}\right)\right)$, i.e., for general forms of degree 6 and 8 in three variables. See also [7], for general criteria of identifiability for symmetric tensors.

Another consequence of Theorem 3.1 is described in Theorem 3.8. Given a minimal decomposition with $r$ addenda, then for any integer $r^{\prime}$ such that $r \leq r^{\prime}<-1+\prod\left(n_{i}+1\right)$, it is always possible to find a non-redundant decomposition with $r^{\prime}$ addenda. We notice that 
this happens to be false for symmetric decompositions of a symmetric tensor, even in the case of $S^{d}\left(\mathbb{C}^{2}\right)$ with $d \geq 4$ (cf. Sylvester theorem in, e.g., [3,9]).

In Sect. 4 , we focus on the identifiability of a minimal decomposition, meaning the uniqueness of a decomposition of a tensor $T$ with exactly $\operatorname{rk}(T)$ addenda. The main result of this section is Theorem 4.6 (that is again a consequence of Theorem 3.1), where we point out a sufficient condition for a non-redundant decomposition to be minimal and unique. Again we compare our result with Kruskal's bound ([15]). Since Kruskal's bound is sharp ([11,18]) and since our geometric assumptions are weaker, and then easier to verify, than Kruskal's ones, we cannot hope to produce applications outside Kruskal's numerical range. There are few cases in which our the numerical range of application matches with Kruskal's range. One of them, e.g., is given by tensors of type $3 \times 2 \times 2 \times 2$.

Let us spend some words on the effectiveness of our criteria for minimality and identifiability. They often require the computation of the dimension $h^{1}$ of a cohomology group of the ideal sheaf of a projection of the inverse image $A$ of a decomposition of the tensor $T$. The computation is a simple algorithm of elementary linear algebra, once one knows $A$. In practice, we require that the projection of $A$ is linearly independent in its Segre embedding, which can be computed straightforwardly from the coordinates of the points of $A$ and hence from the decomposition of $T$. If one does not know a decomposition, only facts about generic tensors $T$ can be derived from our criteria. Let us point out that there are several applications in which the coordinates of the points of $A$ are known. For instance, this can be assumed, as mentioned before, either when one wants to compute the rank of a block tensor, or when one wants to construct examples of tensors of given rank. We also notice the most famous criterion for the study of the identifiability of tensors, namely the Kruskal's criterion, applies only if one knows the Kruskal's rank of a decomposition, which can be computed only if one knows the coordinates of the points of $A$.

\section{Notation and preliminaries}

For a subscheme $Z \subset \mathbb{P}^{m}$, we indicate with $\langle Z\rangle$ the linear span of $Z$ and with $\operatorname{deg}(Z)$ its length (when it is finite). If $Z$ is finite and reduced, we indicate with $\sharp Z$ the cardinality of $Z$.

For any product of projective spaces $\mathbb{P}^{n_{1}} \times \cdots \times \mathbb{P}^{n_{k}}$ call $v$ the Segre map

$$
v: \mathbb{P}^{n_{1}} \times \cdots \times \mathbb{P}^{n_{k}} \rightarrow \mathbb{P}^{M}, \quad M=-1+\prod\left(n_{i}+1\right) .
$$

In order to have a more compact notation, we will always write

$$
Y:=\mathbb{P}^{n_{1}} \times \cdots \times \mathbb{P}^{n_{k}}
$$

for the abstract product, and

$$
X:=v(Y) \subset \mathbb{P}^{M}
$$

for the Segre variety.

For any $i=1, \ldots, k$, call $\pi_{i}$ the projection of $\mathbb{P}^{n_{1}} \times \cdots \times \mathbb{P}^{n_{k}}$ to the $i$ th factor.

We can generalize this notation by setting, for any collection of sub-indices $\mathbf{u}=$ $\left\{u_{1}, \ldots, u_{i}\right\} \subset\{1, \ldots, k\}$,

$$
\pi_{\mathbf{u}}=\text { projection to the product of the factors } u_{1}, \ldots, u_{i} \text {. }
$$

In particular $\pi_{\{1, \ldots, i\}}$ is the projection to the product of the first $i$ factors.

For any subset $\mathbf{u}=\left\{u_{1}, \ldots, u_{i}\right\} \subset\{1, \ldots, k\}$, we will denote with $\mathcal{O}^{\mathbf{u}}(1, \ldots, 1)$ the line bundle on $\mathbb{P}^{n_{1}} \times \cdots \times \mathbb{P}^{n_{k}}$ pull back of the hyperplane bundle on $v\left(\pi_{\mathbf{u}}(Y)\right)$. The line 
bundle $\mathcal{O}^{\{1, \ldots, k\}}(1, \ldots, 1)$ is the pull back of the hyperplane bundle in the Segre embedding of $\mathbb{P}^{n_{1}} \times \cdots \times \mathbb{P}^{n_{k}}$.

For any subset $Z$ of $\mathbb{P}^{n_{1}} \times \cdots \times \mathbb{P}^{n_{k}}$ we will write consequently

$$
\mathcal{I}_{Z}^{\mathbf{u}}(1, \ldots, 1):=\mathcal{I}_{Z} \otimes \mathcal{O}^{\mathbf{u}}(1, \ldots, 1), \quad \mathcal{O}_{Z}^{\mathbf{u}}(1, \ldots, 1):=\mathcal{O}_{Y} \otimes \mathcal{O}^{\mathbf{u}}(1, \ldots, 1) .
$$

Notice that the dimension $h^{0}\left(\mathcal{I}_{Z}^{\mathbf{u}}(1, \ldots, 1)\right)$ corresponds then to the co-dimension of the linear span of $v\left(\pi_{\mathbf{u}}(Z)\right)$.

Then $h^{1}\left(\mathcal{I}_{Z}^{\mathbf{u}}(1, \ldots, 1)\right)$ is the difference between the degree of the scheme $v\left(\pi_{\mathbf{u}}(Z)\right)$ and the affine dimension of its span. Clearly once $h^{0}\left(\mathcal{I}_{Z}^{\mathbf{u}}(1, \ldots, 1)\right)$ is known, then also $h^{1}\left(\mathcal{I}_{Z}^{\mathbf{u}}(1, \ldots, 1)\right)$ is known.

Obviously, if $Z$ is a zero-dimensional scheme and $h^{1}\left(\mathcal{I}_{Z}^{\mathbf{u}}(1, \ldots, 1)\right)=0$, then also $h^{1}\left(\mathcal{I}_{Z}^{\mathbf{u}^{\prime}}(1, \ldots, 1)\right)=0$ for all $\mathbf{u}^{\prime} \supseteq \mathbf{u}$.

We will say that a finite subset $S \subset \mathbb{P}^{n_{1}} \times \cdots \times \mathbb{P}^{n_{k}}$ has different coordinates if, for all $i=1, \ldots, k$, the projection $\pi_{i}$ to the $i$ th factor is an embedding of $S$ into $\mathbb{P}^{n_{i}}$.

We will need the process of residuation with respect to a divisor. We can state this process in complete generality for any projective variety $X$. For any zero-dimensional scheme, $Z \subset X$ and for any effective divisor $D$ on $X$ the "residue of $Z$ w.r.t. $D$ " is the scheme $\operatorname{Res}_{D}(Z)$ defined by the ideal sheaf $\mathcal{I}_{Z}: \mathcal{I}_{D}$, where $\mathcal{I}_{Z}$ and $\mathcal{I}_{D}$ are the ideal sheaves of $Z$ and $D$, respectively. The multiplication by local equations of $D$ defines the exact sequence of sheaves:

$$
0 \rightarrow \mathcal{I}_{\operatorname{Res}_{D}(Z)}(-D) \rightarrow \mathcal{I}_{Z} \rightarrow \mathcal{I}_{D \cap Z, D} \rightarrow 0
$$

where the rightmost sheaf is the ideal sheaf of $D \cap Z$ in $D$.

We will identify elements $T \in \mathbb{P}^{M}$, which is the space of embedding of $Y=\mathbb{P}^{n_{1}} \times \cdots \times \mathbb{P}^{n_{k}}$, as tensors of type $\left(n_{1}+1\right) \times \cdots \times\left(n_{k}+1\right)$ (modulo scalars).

Definition 2.1 A finite reduced subset $S \subset Y=\mathbb{P}^{n_{1}} \times \cdots \times \mathbb{P}^{n_{k}}$ is a decomposition of $T \in \mathbb{P}^{M}$ if $T \in\langle v(S)\rangle$ (with an abuse of notation sometime we will say that also $v(S) \subset X$ is a decomposition of $T$ ). If moreover $T \notin\left\langle v\left(S^{\prime}\right)\right\rangle$ for any $S^{\prime} \subsetneq S$, the decomposition $S$ is said to be not-redundant. Finally, if $\sharp S=\min \left\{\sharp S^{\prime} \mid S^{\prime} \subset Y\right.$ and $\left.T \in\left\langle v\left(S^{\prime}\right)\right\rangle\right\}$ then $S$ is called a minimal decomposition of $T$.

Remark 2.2 Clearly "not-redundant" does not imply "minimal". As we will detail in Theorem 3.8 , it is always possible to build a non-minimal non-redundant decomposition.

Our target is to study the identifiability of a tensor $T \in \mathbb{P}^{M}$ (i.e., when $T$ has only one minimal decomposition) by means of the knowledge of the numbers $h^{0}\left(\mathcal{I}_{A}^{\mathbf{u}}\right)$, for all $\mathbf{u} \subset\{1, \ldots, k\}$, where $A \subset Y$ is a finite set which corresponds to a decomposition of $T$.

We will use the following notions for the rank of $T$ (see $[4,5,16]$ for cactus rank and cactus variety definitions).

Definition 2.3 The $\operatorname{rank}, \operatorname{rk}(T)$, of $T$ is the minimum $r$ for which there exists a minimal decomposition of $T$.

The cactus rank of $T$ is the minimum $r$ for which there exists a zero-dimensional subscheme $\Gamma \subset X$ with $\operatorname{deg}(\Gamma)=r$ and $T \in\langle\Gamma\rangle$.

Clearly:

rank of $(T) \geq$ cactus rank of $(T)$. 
In analogy to the rank case, we will say that a zero-dimensional scheme $Z \subset Y$ is a "minimal cactus decomposition" of $T \in \mathbb{P}^{M}$ if $Z$ is of minimal degree among the zero-dimensional schemes $Z^{\prime} \subset Y$ such that $T \in\left\langle\nu\left(Z^{\prime}\right)\right\rangle$.

If $\sigma_{r}$ is the $r$-secant variety of $X$, then all tensors of rank $r$ belong to $\sigma_{r}$.

For any tensor $T$ of rank $r$, let $\mathcal{S}(T)$ denote the set of all (reduced) finite subsets $S \in Y$ of cardinality $r$ such that $T \in\langle v(S)\rangle$. Of course for all $S \in \mathcal{S}(T)$ the image $v(S)$ is linearly independent, for otherwise $T$ is contained in the span of a subset of cardinality $r^{\prime}<r$; thus, it has rank smaller than $r$.

A tensor $T$ is identifiable when $\mathcal{S}(T)$ is a singleton. The abstract product $Y=\mathbb{P}^{n_{1}} \times \cdots \times$ $\mathbb{P}^{n_{k}}$ is "generically identifiable in rank $r$ " if the general $T \in \sigma_{r}$ is identifiable.

The main tool for our analysis of the identifiability of a tensor $T \in \mathbb{P}^{M}$ relies in the following proposition that is an immediate consequence of [2, Lemma 1] if we consider the residue exact sequence of $A \cup B$ cut by a linear space containing $A$.

Proposition 2.4 Consider linearly independent zero-dimensional schemes $A, B \subset Y$. Then the linear spans of the images $v(A), v(B)$ in the Segre map satisfy

$$
\operatorname{dim}(\langle v(A)\rangle \cap\langle v(B)\rangle)=\operatorname{dim}(\langle v(A \cap B)\rangle)+h^{1}\left(\mathcal{I}_{A \cup B}^{\{1, \ldots, k\}}(1, \ldots, 1)\right) .
$$

\section{Rank}

If we know a decomposition $T=T_{1}+\cdots+T_{r}$ of $T$ in terms of tensors $T_{i}$ of rank 1 , in general we cannot directly conclude that $r$ is the rank of $T$.

Our analysis will prove that, for small values of $r$, the rank of $T$ is $r$ provided that the summands correspond to points in a suitably general geometric position. We will give a criterion to find a lower bound for the cactus rank (and therefore also for the rank).

Theorem 3.1 Fix a partition $E \sqcup F=\{1, \ldots, k\}$ of the $k$ factors of the abstract product $Y=\mathbb{P}^{n_{1}} \times \cdots \times \mathbb{P}^{n_{k}}$, i.e., $E=\left\{a_{1}, \ldots, a_{k-h}\right\}$ and $F=\left\{b_{1}, \ldots, b_{h}\right\}$ for some fixed $0<h<k$. Let $M_{F}:=\prod_{i=1}^{h}\left(n_{b_{i}}+1\right)$ be the affine dimension of the ambient space of the Segre embedding of the $F$-factors $Y_{F}:=\mathbb{P}^{b_{1}} \times \cdots \times \mathbb{P}^{b_{h}}$. Now fix $0<c<M_{F}$ and let $A \subset Y$ be a zero-dimensional scheme which satisfies $h^{1}\left(\mathcal{I}_{A}^{E}(1, \ldots, 1)\right)=0$ and $h^{0}\left(\mathcal{I}_{A}^{F}(1, \ldots, 1)\right)<M_{F}-c$. Take any $T \in\langle v(A)\rangle$ such that $T \notin\left\langle v\left(A^{\prime}\right)\right\rangle$ for any $A^{\prime} \subsetneq A$. Then there are no zero-dimensional schemes $B \subset Y$ with $\operatorname{deg}(B) \leq c$ such that $T \in\langle\nu(B)\rangle$.

Proof Notice that the Segre embedding of the projection $\pi_{F}$ maps $Y$ to $\mathbb{P}^{M_{F}-1}$.

Since $h^{1}\left(\mathcal{I}_{A}^{E}(1, \ldots, 1)\right)=0$, we have $h^{1}\left(\mathcal{I}_{A}^{\{1, \ldots, k\}}(1, \ldots, 1)\right)=0$. The condition $h^{0}\left(\mathcal{I}_{A}^{F}(1, \ldots, 1)\right)<M_{F}-c$ implies that $\operatorname{deg}(A)>c$. Assume that the theorem fails and take $B \subset Y$ with $\operatorname{deg}(B) \leq c$ and $T \in\langle v(B)\rangle$. Since $\operatorname{deg}(A)>\operatorname{deg}(B)$ and $T \notin\left\langle v\left(A^{\prime}\right)\right\rangle$ for any $A^{\prime} \subsetneq A$, we have $B \nsubseteq A$. Moreover $h^{1}\left(\mathcal{I}_{A \cup B}^{\{1, \ldots, k\}(1, \ldots, 1)}\right)>0$, by Proposition 2.4. More precisely, since $T \in\langle v(B)\rangle$ and $T \notin\left\langle v\left(A^{\prime}\right)\right\rangle$ for any $A^{\prime} \subsetneq A$, we have

$$
h^{1}\left(\mathcal{I}_{A \cup B}^{\{1, \ldots, k\}}(1, \ldots, 1)\right)>h^{1}\left(\mathcal{I}_{A^{\prime} \cup B}^{\{1, \ldots, k\}}(1, \ldots, 1)\right)
$$

for all $A^{\prime} \subset A$ with $A \cup B \neq A^{\prime} \cup B$.

Since $\operatorname{deg}(B) \leq c<M_{F}$, we have $h^{0}\left(\mathcal{I}_{B}^{F}(1, \ldots, 1)\right)>0$. Take a general divisor $D \in\left|\mathcal{I}_{B}^{F}(1, \ldots, 1)\right|$. In other words, $D$ is the inverse image in $Y$ of a hyperplane in the Segre embedding of $Y_{F}$ and $B \subset D$. Since $h^{0}\left(\mathcal{I}_{A}^{F}(1, \ldots, 1)\right)<M_{F}-c \leq h^{0}\left(\mathcal{I}_{B}^{F}(1, \ldots, 1)\right)$, 
then $A \nsubseteq D$, so that $(D \cap A) \cup B$ is strictly contained in $A \cup B$. Hence by (3) we get $h^{1}\left(\mathcal{I}_{(D \cap A) \cup B}^{\{1, \ldots, k\}}(1, \ldots, 1)\right)<h^{1}\left(\mathcal{I}_{A \cup B}^{\{1, \ldots, k\}}(1, \ldots, 1)\right)$. The residual exact sequence (2) applied to $D$ gives $h^{1}\left(\mathcal{I}_{\operatorname{Res}_{D}(A \cup B)}^{E}(1, \ldots, 1)\right)>0$. Since $\operatorname{Res}_{D}(A \cup B) \subseteq A$, we get a contradiction.

Observe that the condition $h^{1}\left(\mathcal{I}_{A}^{E}(1, \ldots, 1)\right)=0$ can be satisfied only when $\operatorname{deg}(A) \leq$ $\prod_{i=1}^{k-h}\left(n_{a_{i}}+1\right)$, the affine dimension of the ambient space of the Segre embedding of the $E$-factors $Y_{E}=\mathbb{P}^{a_{1}} \times \cdots \times \mathbb{P}^{a_{k-h}}$.

We can rephrase Theorem 3.1 to produce results on the rank of tensors.

Corollary 3.2 With the previous notation, if $T$ sits in the linear span of a scheme $A \subset Y$ which satisfies the assumptions of Theorem 3.1, then the cactus rank of $T$ is a least $c+1$. Hence also the rank of $T$ cannot be smaller than $c+1$.

Corollary 3.3 Let $A \subset Y$ be a zero dimensional scheme of $\operatorname{deg}(A)=c+1$ (resp. a finite set with $\sharp(A)=c+1)$. With the Notation of Theorem 3.1, assume that $h^{1}\left(\mathcal{I}_{A}^{E}(1, \ldots, 1)\right)=0$ and $h^{1}\left(\mathcal{I}_{A}^{F}(1, \ldots, 1)\right)=0$. Then any $T \in\langle v(A)\rangle$ such that $T \notin\left\langle v\left(A^{\prime}\right)\right\rangle$ for any $A^{\prime} \subsetneq A$ has cactus rank (resp. cactus rank and rank) equal to $c+1$.

Proof It is straightforward from Theorem 3.1

Let we point out an application to the case of 3-way tensors.

Proposition 3.4 Consider $k=3$ and let $T$ be a tensor of type $\left(n_{1}+1\right) \times\left(n_{2}+1\right) \times\left(n_{3}+1\right)$, which has a not-redundant decomposition $T=T_{1}+\cdots+T_{r}$, where the $T_{i}$ 's are tensors of rank 1. Identify each $T_{i}$ with a point in $X=v\left(\mathbb{P}^{n_{1}} \times \mathbb{P}^{n_{2}} \times \mathbb{P}^{n_{3}}\right)$ and set $A=\left\{T_{1}, \ldots, T_{r}\right\} \subset X$. Call $A_{E}$ (resp. $\left.A_{F}\right)$ the projection of $A$ to $Y_{E}=\mathbb{P}^{n_{1}} \times \mathbb{P}^{n_{2}}\left(\right.$ resp. $\left.Y_{F}=\mathbb{P}^{n_{3}}\right)$.

Assume that $A$ has different coordinates, $A_{E}$ is linearly independent and $A_{F}$ is contained in no hyperplanes of $\mathbb{P}^{n_{3}}$. Then the rank of $T$ is at least $n_{3}+1$.

Proof In the notation of Theorem 3.1, take $E=\{1,2\}$ and $F=\{3\}$. Then our assumptions on $A_{E}, A_{F}$ imply that $A$ satisfies $h^{1}\left(\mathcal{I}_{A}^{E}(1, \ldots, 1)\right)=h^{0}\left(\mathcal{I}_{A}^{F}(1, \ldots, 1)\right)=0$. Thus $T$ cannot have a decomposition with $M_{F}-1=n_{3}$ summands.

Example 3.5 Kruskal's Theorem for the identifiability of tensors ([15]) provides results similar to the previous proposition for the rank. We notice that the numerical range of application of Proposition 3.4 is sometimes wider than Kruskal's range.

For instance, consider a tensor $T$ of type $3 \times 4 \times 6$ having a decomposition with 6 summands. If the decomposition determines a subset $A$ satisfying the assumptions of Proposition 3.4, we can conclude that the rank of $T$ is 6 . We cannot get the same conclusion directly with Kruskal's Theorem because we are outside Kruskal's numerical range, since $6>(3+4+6-2) / 2$.

The following example should be considered as a step toward the Strassen's Conjecture on the rank of a block tensor (see [19]).

Example 3.6 Proposition 3.4 can give results on the rank of a sum of tensors, when we have some information on the decompositions of the summands.

For instance, consider again tensors of type $3 \times 4 \times 6$ and take a tensor $T$ which is the sum $T=T^{\prime}+T^{\prime \prime}$ of two tensors of rank 3 . Consider a decomposition of $T^{\prime}$ (resp. $T^{\prime \prime}$ ) in a sum of three tensors of rank 1 and call $S^{\prime}$ (resp. $S^{\prime \prime}$ ) the set of cardinality 3 in the product $\mathbb{P}^{2} \times \mathbb{P}^{3} \times \mathbb{P}^{5}$ determined by the decomposition. 
If the set $S=S^{\prime} \cup S^{\prime \prime}$ has cardinality 6 and satisfies the assumptions of Proposition 3.4 (i.e., both $\pi_{\{1,2\}}(\nu(S))$ and $\pi_{\{3\}}(\nu(S))$ are linearly independent), then we can conclude that the rank of $T$ is 6 .

We show below that, on the contrary, if we increase the cardinality of a decomposition, we can always construct new non-redundant decompositions of a tensor $T$.

Example 3.7 Fix $P \in Y$ and write $P=\left(p_{1}, \ldots, p_{k}\right)$ with $p_{i} \in \mathbb{P}^{n_{i}}$. Assume $n_{i}>0$. Take two points $b_{i}, c_{i} \in \mathbb{P}^{n_{i}}$ such that $p_{i} \neq b_{i}, c_{i}$ but $p_{i}$ is contained in the line of $\mathbb{P}^{n_{i}}$ spanned by $b_{i}$ and $c_{i}$. Let $O_{i}:=\left(u_{1}, \ldots, u_{k}\right), Q_{i}:=\left(v_{1}, \ldots, v_{k}\right)$ be the points of $Y$ with $u_{j}=v_{j}$ for all $j \neq i, u_{i}=b_{i}$ and $v_{i}=c_{i}$. We have $v(P) \in\left\langle\left\{v\left(O_{i}\right), v\left(Q_{i}\right)\right\}\right\rangle$, and of course $v(P) \notin\left\langle v\left(S^{\prime}\right)\right\rangle$ for any $S^{\prime} \subsetneq\left\{O_{i}, Q_{i}\right\}$.

We show that indeed the previous construction can yield a non-redundant decomposition. Moreover the following results also show that having found a non-redundant decomposition does not imply that it is a minimal one.

Theorem 3.8 Assume $n_{i}>0$ for at least one $i$. Take a finite set $A \subset X$ of cardinality $r \leq M$, such that $v(A) \subset X$ is linearly independent. Take a general $T \in\langle v(A)\rangle$. Then there exists a non-redundant decomposition $S \subset Y$ of $T$ of cardinality $r+1$.

Proof Fix $P=\left(p_{1}, \ldots, p_{k}\right) \in A$ and take $Q_{1}, O_{1}$ as in Example 3.7, with $i=1$, and with the additional condition that $O_{1}, Q_{1} \notin(A \backslash\{P\})$. We may take $O_{1}$ to be a general point of $\mathbb{P}^{n_{1}} \times\left\{p_{2}\right\} \times \cdots \times\left\{p_{k}\right\}$. Hence we may take $O_{1}=\left(a_{1}, p_{2}, \ldots, p_{k}\right)$, with $a_{1}$ general. Set $A^{\prime}:=A \backslash\{P\}$.

(a) Assume $v\left(O_{1}\right) \notin\langle v(A)\rangle$. This is always possible unless $\langle v(A)\rangle$ contains $\mathbb{P}^{n_{1}} \times\left\{p_{2}\right\} \times$ $\cdots \times\left\{p_{k}\right\}$. Since $P \in A$, this is equivalent to $v\left(Q_{1}\right) \notin\langle v(A)\rangle$. Set $S:=(A \backslash\{P\}) \cup\left\{O_{1}, Q_{1}\right\}$. We have $\sharp(S)=r+1$ and $\langle v(S)\rangle \supseteq\langle v(A)\rangle$ so that $T \in\langle v(S)\rangle$. The set $S$ does not depend on $Q_{1}$, but only on $A$ and $P$. To prove that $S$ satisfies the claim, it is sufficient to prove that for a general $T \in\langle v(A)\rangle$ there is no $S^{\prime} \subsetneq S$ with $T \in\left\langle v\left(S^{\prime}\right)\right\rangle$. It is sufficient to test all subsets of $S$ with cardinality $r$. Take $S^{\prime} \subset S$ with $\sharp\left(S^{\prime}\right)=r$. If $S^{\prime} \supset A^{\prime}$, i.e., if either $S^{\prime}=A^{\prime} \cup\left\{O_{1}\right\}$ or $S^{\prime}=A^{\prime} \cup\left\{Q_{1}\right\}$, then $\left\langle v\left(S^{\prime}\right)\right\rangle \cap\langle v(A)\rangle=\left\langle v\left(A^{\prime}\right)\right\rangle$, because $\left\{v\left(O_{1}\right), v\left(Q_{1}\right)\right\} \cap\langle v(A)\rangle=\emptyset$. Thus $T$ cannot stay in $\left\langle v\left(S^{\prime}\right)\right\rangle$ because it cannot stay in $\left\langle v\left(A^{\prime}\right)\right\rangle$ for a proper subset $A^{\prime}$ of $A$. Hence $S^{\prime} \nsupseteq A^{\prime}$. Since $\sharp\left(S^{\prime}\right)=r$, we have $S^{\prime}=A^{\prime \prime} \cup\left\{O_{1}, Q_{1}\right\}$ with $A^{\prime \prime} \subset A^{\prime}$ and $\sharp\left(A^{\prime \prime}\right)=r-2$. If $T \in\left\langle v\left(S^{\prime}\right)\right\rangle$, then $\left\langle v\left(S^{\prime}\right)\right\rangle \cap\langle v(A)\rangle \supseteq\left\langle v\left(A^{\prime \prime}\right) \cup\{T\}\right\rangle$. Since $v\left(O_{1}\right) \notin\langle v(A)\rangle$, we get:

$$
\left\langle v\left(S^{\prime}\right)\right\rangle \cap\langle v(A)\rangle=\left\langle v\left(A^{\prime \prime}\right) \cup\{T\}\right\rangle .
$$

The left hand side of this equality does not depend on the choice of $T$. Varying $T \in$ $\langle v(A)\rangle \backslash\left\langle v\left(A^{\prime}\right)\right\rangle$ we get $\left\langle v\left(S^{\prime}\right)\right\rangle \supseteq\langle v(A)\rangle$. Since $\sharp\left(S^{\prime}\right)=\sharp(A)$ and $v(A)$ is linearly independent, we get $\left\langle v\left(S^{\prime}\right)\right\rangle=\langle v(A)\rangle$ and hence $v\left(O_{1}\right) \in\langle v(A)\rangle$, a contradiction.

(b) If $n_{j}=0$ for all $j>1$ we are done. Assume for instance that $n_{2}>0$ and that $\langle v(A)\rangle \supset \mathbb{P}^{n_{1}} \times\left\{p_{2}\right\} \times \cdots \times\left\{p_{k}\right\}$, so that we cannot take $v\left(O_{1}\right) \notin\langle v(A)\rangle$. Since $v(P) \in$ $\left\langle\left\{v\left(O_{1}\right), v\left(Q_{1}\right)\right\}\right\rangle$, we get $\left\langle v\left(\left\{O_{1}\right\} \cup A^{\prime}\right)\right\rangle=\langle v(A)\rangle$. Replace thus $A$ with $A^{\prime} \cup\left\{O_{1}\right\}$ and $P$ by $O_{1}$. Then take the construction of Example 3.7 with $i=2$. If the new general point $\mathrm{O}_{2}$ satisfies $v\left(\mathrm{O}_{2}\right) \notin\langle v(A)\rangle$, then we conclude as in step (a). Otherwise we have $\langle v(A)\rangle \supset$ $\mathbb{P}^{n_{1}} \times \mathbb{P}^{n_{2}} \times\left\{p_{3}\right\} \times \cdots \times\left\{p_{k}\right\}$ Then continue with $i=3$ in the construction of Example 3.7, and so on. After at most $k$ steps, we must conclude by step (a), because $\langle A\rangle$ cannot contain the whole product $Y$, since $\sharp(A) \leq M$.

Remark 3.9 Take $P=\left(p_{1}, \ldots, p_{k}\right), i \in\{1, \ldots, k\}$ with $n_{i}>0$ and $b_{i}, c_{i}$ as in Example 3.7. Notice that in the previous proof we can choose for $c_{i}$ any point (different from $b_{i}$ ) in the 
line spanned by $b_{i}, p_{i}$. Thus, in Theorem 3.8 we get a positive-dimensional family of sets $S \subset Y$ such that $\sharp(S)=r+1, T \in\langle\nu(S)\rangle$ and $T \notin\left\langle\nu\left(S^{\prime}\right)\right\rangle$ for any $S^{\prime} \subsetneq S$.

We end this section with a discussion on some consequence of Theorem 3.1 on symmetric tensors.

Assume $n:=n_{1}=\cdots=n_{k}$ so that $Y$ is a product of $k$ copies of $\mathbb{P}^{n}$. The Segre map restricted to the diagonal $\Delta \subset Y$ can be identified with the Veronese map $v_{k}: \mathbb{P}^{n} \rightarrow \mathbb{P}^{D}$ where $D+1=\left(\begin{array}{c}k+n \\ n\end{array}\right)$. The space $\mathbb{P}^{D}$ parameterizes symmetric tensors $T$, which in turn can be identified with homogeneous polynomials (forms) of degree $k$ in $n+1$ variables. The symmetric rank is the minimum $r$ for which there exists a finite subset $A \subset \mathbb{P}^{n}$ of cardinality $r$ with $T \in\left\langle v_{k}(A)\right\rangle$.

A conjecture raised in [10] and well known as Comon's conjecture predicts that the symmetric rank of a symmetric tensor $T$ always coincides with the rank of $T$ as a normal tensor in the span of $v(Y)$. The first counterexample to the complete validity of that conjecture is due to Shitov [17], and it is a polynomial of degree 3 in 800 variables that has symmetric rank strictly bigger than 903 which is its rank.

The following corollary of Theorem 3.1 implies that, if some assumptions on a minimal symmetric decomposition $A$ of a symmetric tensor $T$ are satisfied, that the symmetric rank of $T$ coincides with its rank (and cactus rank).

Corollary 3.10 With the previous notation, consider a zero-dimensional scheme $A \subset \mathbb{P}^{n}$ of degree $\operatorname{deg}(A)=c+1$. Call $\mathcal{J}_{A}$ the ideal sheaf of $A$ in $\mathbb{P}^{n}$ and assume that $h^{1}\left(\mathcal{J}_{A}(e)\right)=0$ for some $e \leq k / 2$. Take $T \in\left\langle v_{k}(A)\right\rangle$ such that $T \notin\left\langle v_{k}\left(A^{\prime}\right)\right\rangle$ for any $A^{\prime} \subsetneq A$. Then $T$ has cactus rank equal to $c+1$. If $A$ is reduced (i.e., it is a finite set of points), then $T$ has also rank $c+1$.

Proof Consider any subset $E \subset\{1, \ldots, k\}$ of cardinality $e$ and take $F=\{1, \ldots, k\} \backslash E$. Notice that $f:=\sharp F \geq e$, so that also $h^{1}\left(\mathcal{J}_{A}(f)\right)=0$. This implies that, considering $A$ as a subset of $\Delta \subset Y$, in the notation of Theorem 3.1, we have $h^{1}\left(\mathcal{I}_{A}^{E}(1, \ldots, 1)\right)=0$ and $h^{0}\left(\mathcal{I}_{A}^{F}(1, \ldots, 1)\right)<M_{F}-c$. The claim follows from Theorem 3.1.

We show that Corollary 3.10 provides new range where Comon's conjecture holds: such a range is sometimes even larger than the ones considered in previous works devoted to prove the validity of Comon's conjecture (e.g., [12,20]); Shitov's example [17] is out of our range.

Remark 3.11 Assume $k=2 e$ even. Then by Corollary 3.10, the Comon's conjecture holds for symmetric tensors $T$ having a minimal symmetric decomposition $A$ with $h^{1}\left(\mathcal{J}_{A}(e)\right)=0$.

The condition $h^{1}\left(\mathcal{J}_{A}(e)\right)=0$ holds for general subsets $A \subset \mathbb{P}^{n}$, as soon as $c+1=\sharp A$ satisfies

$$
c+1 \leq r_{0}:=\left(\begin{array}{c}
n+e \\
e
\end{array}\right) .
$$

So Comon's conjecture holds for general tensors whose symmetric rank $c+1$ is bounded by $r_{0}$.

When $k=2 e+1$ is odd, a similar conclusion holds with $r_{0}:=1+\left(\begin{array}{c}n+e \\ e\end{array}\right)$.

Clearly, this remark does not apply to [17] since in that case it is $e=1$ and $903>$ $\left(\begin{array}{c}800-1+1 \\ 1\end{array}\right)=800$. Moreover, Shitov's example is far from being a general one.

Example 3.12 After the Alexander-Hirschowitz classification of defective Veronese varieties ([1]), a general symmetric tensor in the span of $v_{k}\left(\mathbb{P}^{n}\right)$ is known to have symmetric rank

$$
r_{g}=\left\lceil\frac{\left(\begin{array}{c}
n+k \\
k
\end{array}\right)}{n+1}\right\rceil
$$


except for a list of few exceptional cases.

In general $r_{g}$ is bigger than our bound $r_{0}$ of Remark 3.10, since, for fixed $e, r_{g}$ grows asymptotically as $\left(2^{n} e^{n}\right) /(n+1)$ ! while $r_{0}$ grows like $e^{n} / n !$.

Nevertheless, there are cases in which $r_{g}$ and $r_{0}$ coincide. This happens for $(k, n)=(6,2)$ or $(k, n)=(8,2)$. Since these two cases are not in the list of exceptional Veronese varieties, we can conclude that Comon's Conjecture holds for general forms of degree 6 and degree 8 in 3 variables.

Example 3.13 Take $(k, n)=(4,3)$. Then Remark 3.11 tells us that the Comon's conjecture holds for general symmetric tensors in the span of $v_{4}\left(\mathbb{P}^{3}\right)$ with a decomposition with 10 summands. On the other hand, in this case the number $r_{g}$ is 9 , smaller than $r_{0}$.

Indeed $(k, n)=(4,3)$ is in the list of exceptional cases in the Alexander-Hirschowitz theorem, so that a general form of degree 4 in $\mathbb{P}^{3}$ has symmetric rank $10>r_{g}$. Thus Comon's conjecture also holds for such general forms.

In some sense, Theorem 3.1 provides a new heuristic reason why the case $(k, n)=(4,3)$ is exceptional: a general tensor with a non-redundant decomposition with 10 summands cannot have a decomposition with 9 summands. A similar remark holds for the other exceptional cases of even degree: quadrics in any $\mathbb{P}^{n}$ and quartics in $\mathbb{P}^{2}$ and $\mathbb{P}^{4}$.

\section{Identifiability}

In order to get results on the identifiability of a tensor $T$, we need to refine the previous analysis, and we are going to do that in this section.

We will need the following terminology for the Segre function of a finite subset of the product, introduced in [8].

Definition 4.1 For any set of points $S \subset Y$, the Segre function $\mathrm{SF}_{S}:\{1, \ldots, k\} \rightarrow \mathbb{N}$ is defined by:

$$
\mathrm{SF}_{S}(i)=1+\text { the dimension of the linear span of } v\left(\pi_{\{1, \ldots, i\}}(S)\right) \text {. }
$$

Remark that the knowledge of the sequence $h^{0}\left(\mathcal{I}_{S}^{\{1, \ldots, i\}}(1, \ldots, 1)\right), i=1, \ldots, k$, is equivalent to the knowledge of the Segre function $\mathrm{SF}_{S}$.

More precisely, the definition of Segre function depends on the ordering of the factors of the product. The knowledge of $h^{0}\left(\mathcal{I}_{S}^{\mathbf{u}}(1, \ldots, 1)\right)$, for all possible $\mathbf{u} \subset\{1, \ldots, k\}$, is equivalent to the knowledge of the Segre functions of $S$ under all possible re-arrangements of the factors.

Let us recall the following definition for a minimal dependent set of point (the same can be found in [8, Definition 2.9], while where in [13, Chap.7 Sec.1] a minimal dependent set of points is called a circuit).

Definition 4.2 A set of points $S \subset \mathbb{P}^{m}$ is minimally dependent, if $S$ is linearly dependent, but any proper subset of $S$ is linearly independent.

We need now a list of results on the cohomology of $\mathcal{I}_{S}(1, \ldots, 1)$, for finite sets $S$ in a product of projective spaces.

Definition 4.3 We say that a finite subset $S \subset Y$ is concise if for all indexes $i$ the set $\pi_{i}(S)$ spans $\mathbb{P}^{n_{i}}$, i.e., there are no hyperplanes $H \subset \mathbb{P}^{n_{i}}$ such that

$$
S \subset \mathbb{P}^{n_{1}} \times \cdots \times \mathbb{P}^{n_{i-1}} \times H \times \mathbb{P}^{n_{i+1}} \times \cdots \times \mathbb{P}^{n_{k}} .
$$


We say that $T \in \mathbb{P}^{M}$ is concise if there are no non-concise subsets $A \subset v(Y)$ such that $T \in\langle A\rangle$.

Notice that if $S \subset Y$ is concise, then necessarily $\sharp S>\max \left\{n_{i}\right\}$.

The two results below are mainly an extension to the non-symmetric case of results in [6] and [2].

Lemma 4.4 Fix a finite set $S \subset Y=\mathbb{P}^{n_{1}} \times \cdots \times \mathbb{P}^{n_{k}}$ such that $x:=\sharp(S) \leq k+1$, $h^{1}\left(\mathcal{I}_{S}^{\{1, \ldots, k\}}(1, \ldots, 1)\right)>0$ and $h^{1}\left(\mathcal{I}_{S^{\prime}}^{\{1, \ldots, k\}}(1, \ldots, 1)\right)=0$ for each $S^{\prime} \subsetneq S$. Then there is $E \subset\{1, \ldots, k\}$ such that $\sharp(E)=k+2-x$ and $\sharp\left(\pi_{i}(S)\right)=1$ for all $i \in E$.

Proof The lemma is trivial if $x=2$, because $\mathcal{O}_{Y}^{\{1, \ldots, k\}}(1, \ldots, 1)$ is very ample and so the assumptions of the lemma are never satisfied in this case. Thus we may assume $x>2$ and use induction on the integer $x$.

Now assume $x=3$. By assumption $v(S)$ is contained in a line $L \subset X$, i.e., there is a curve $D \subset Y$ such that $v(D)$ is a line, i.e., $\operatorname{deg}\left(\mathcal{O}_{D}(1, \ldots, 1)\right)=1$. We have $\operatorname{deg}\left(\mathcal{O}_{D}(1, \ldots, 1)\right)=$ $\sum_{i=1}^{k} \operatorname{deg}\left(\pi_{i \mid D}\right)$. Hence there is $E \subset\{1, \ldots, k\}$ such that $\sharp(E)=k-1$ and $\operatorname{deg}\left(\pi_{i \mid D}\right)=0$ for all $i \in E$, i.e., $\pi_{i}(D)$ is a point. Thus $\pi_{i}(S)$ is a point for all $i \in E$.

Thus we assume $x>3$ (and so $k \geq 3$ ).

Assume $\sharp\left(\pi_{1}(S)\right) \geq 2$, so that the Segre function of $S$ satisfies $\operatorname{SF}_{S}(1) \geq 2$. Notice that the assumptions on $S$ imply that $\mathrm{SF}_{S}(k)=x-1$. Take points $P=\left(a_{1}, \ldots, a_{k}\right)$, $Q=\left(b_{1}, \ldots, b_{k}\right) \in S$ with $P \neq Q$. Since $h^{1}\left(\mathcal{I}_{S^{\prime}}^{\{1, \ldots, k\}}(1, \ldots, 1)\right)=0$ for all $S^{\prime} \subsetneq S$ and $x>3$, we may find $A \supset\{P, Q\}$ with $\sharp(A)=x-1$ and $h^{1}\left(\mathcal{I}_{A}^{\{1, \ldots, k\}}(1, \ldots, 1)\right)=0$. Since $x \leq k+1$, there is a minimal integer $i \in\{2, \ldots, k\}$ such that $\operatorname{SF}_{S}(i-1)=\operatorname{SF}_{S}(i)$. By [8, Proposition 2.5] for every minimally dependent $S^{\prime} \subseteq S$ with respect to the line bundle $\mathcal{O}^{\{1, \ldots, i\}}(1, \ldots, 1)$, we have $\sharp\left(\pi_{i}\left(S^{\prime}\right)\right)=1$. Since $i \geq 2$ and $P \neq Q$, we have $h^{1}\left(\mathcal{I}_{\{P, Q\}}^{\{1, \ldots, i\}}(1, \ldots, 1)\right)=0$. Hence we may find a minimally dependent set containing $\{P, Q\}$. Thus $a_{i}=b_{i}$. Take any $C=\left(c_{1}, \ldots, c_{k}\right) \in S \backslash\{P, Q\}$. If $c_{1} \neq a_{1}$, we may take minimally dependent $S^{\prime \prime} \subseteq S$ with respect to the line bundle $\mathcal{O}^{\{1, \ldots, i-1\}}(1, \ldots, 1)$ containing $\{P, C\}$ and hence $c_{i}=a_{i}$. If $c_{1}=a_{1}$, we may take minimally dependent $S^{\prime \prime} \subseteq S$ with respect to the line bundle $\mathcal{O}^{\{1, \ldots, i-1\}}(1, \ldots, 1)$ containing $\{Q, C\}$ and hence $c_{i}=b_{i}=a_{i}$. Thus $\pi_{i}(S)=\left\{a_{i}\right\}$. The lemma is now proved when $x=k+1$, by setting $E=\{i\}$.

If $x<k+1$, we apply the same proof to the projection $\pi_{\mathbf{u}}(X)$ where $\mathbf{u}=\{1, \ldots, k\} \backslash\{i\}$ and conclude by descending induction on the integer $k+1-x$.

Lemma 4.5 Fix a finite set $S \subset Y$ such that $x:=\sharp(S) \leq k+n_{1},\left\langle\pi_{1}(S)\right\rangle=\mathbb{P}^{n_{1}}$, $h^{1}\left(\mathcal{I}_{S}^{\{1, \ldots, k\}}(1, \ldots, 1)\right)>0$ and $h^{1}\left(\mathcal{I}_{S^{\prime}}^{\{1, \ldots, k\}}(1, \ldots, 1)\right)=0$ for each $S^{\prime} \subsetneq S$. Then there is $E \subset\{2, \ldots, k\}$ such that $\sharp(E)=k+2-x$ and $\sharp\left(\pi_{i}(S)\right)=1$ for all $i \in E$.

Proof Take $n_{1}+1$ points $A_{1}, \ldots, A_{n_{i}+1}$, say $A_{j}=\left(a_{j, 1}, \ldots, a_{j, k}\right), 1 \leq j \leq n_{1}+1$, with the property that the set $\left\{a_{1,1}, \ldots, a_{n_{1}+1,1}\right\}$ spans $\mathbb{P}^{n_{1}}$. Then just repeat the proof of Lemma 4.4 .

Now, with the aid of Proposition 2.4, we are ready to prove the following theorem where we explicit a sufficient condition for a non-redundant decomposition to be minimal and unique.

Theorem 4.6 Fix an element $T \in \mathbb{P}^{M}$ and a non-redundant decomposition $S \subset Y$ of $T$ and let $\sharp(S)=r$. If $m:=\max \left\{n_{1}, \ldots, n_{k}\right\}$, then 
(a) If $2 r \leq k+m$, then the rank of $T$ is $r$.

(b) If moreover $2 r<k+m$, then $\mathcal{S}(T)=\{S\}$, i.e., $T$ is identifiable.

Proof By permuting the factors of $Y$, we may assume that $n_{1}=m$.

The assumptions on $T$ imply in particular that $v(S)$ is linearly independent, i.e., $h^{1}\left(\mathcal{I}_{S}^{\{1, \ldots, k\}}(1, \ldots, 1)\right)=0$. Let $S^{\prime} \subset Y$ be a minimal decomposition of $T$ such that $\sharp S^{\prime} \leq r$ and $S^{\prime} \neq S$. Then, by Proposition 2.4, $h^{1}\left(\mathcal{I}_{S \cup S^{\prime}}^{\{1, \ldots\}}(1, \ldots, 1)\right)>0$.

Let $\tilde{S} \subseteq S \cup S^{\prime}$ be a minimal subset of $S \cup S^{\prime}$ containing $S$ and such that $h^{1}\left(\mathcal{I}_{\tilde{S}}^{\{1, \ldots, k\}}(1, \ldots, 1)\right)>0$. Since $\tilde{S} \supseteq S$, and $S$ is concise, the set $\pi_{1}(\tilde{S})$ spans $\mathbb{P}^{n_{1}}$. By Lemma 4.5, we have $\sharp(\tilde{S}) \geq k+n_{i}+1$ and hence $\sharp\left(S^{\prime}\right)+r \geq k+m+1$.

If $2 r=k+m$ we get $\sharp\left(S^{\prime}\right) \geq r$. This proves that $T$ has rank $r$. If $2 r<k+m$ we get a contradiction.

Corollary 4.7 Set $m:=\max \left\{n_{1}, \ldots, n_{k}\right\}$. Take a concise $T \in \mathbb{P}^{M}$. If $2 \operatorname{rk}(T)<k+m$, then $T$ is identifiable.

Proof Take $S \in \mathcal{S}(T)$. Since $S \subset Y$ and $T \in\langle v(S)\rangle$, then $X$ is the minimal multiprojective space containing $S$. Then apply part (4.6) of Theorem 4.6.

Remark 4.8 One cannot give a result on the identifiability of tensors without comparing it with the celebrated Kruskal's bound ([15]), which is known to be sharp ([11,18]).

Our condition on the decomposition $S$ of the tensor $T$ is weaker than the condition imposed by Kruskal, which requires to compute the span of any subset of $\pi_{i}(S)$ up to cardinality $n_{i}+1$ (in order to determine the Kruskal's rank), while we only need to check that $\pi_{i}(S)$ generates $\mathbb{P}^{n_{i}}$. Since the Kruskal's rank of the projections of $S$ in principle can be even 1 (when $\pi_{i}$ is not injective), for low values of the rank our result determines the identifiability of $T$ under weaker assumptions.

Of course, as our assumptions are weaker than Kruskal's ones, we cannot give applications outside Kruskal's numerical range. There are few cases in which the numerical range of application of our result matches with Kruskal's range. One of them, e.g., is given by tensors of type $3 \times 2 \times 2 \times 2$.

We notice that tensors of format $3 \times 2 \times 2 \times 2$ and of generic rank 4 are also generically identifiable. This has been discovered in Theorem 1.2 of [14], still by using projections onto different factors.

Next, we show that under some condition on the decomposition $S$ of a tensor $T$, we can prove that any other decomposition $S^{\prime}$ of cardinality smaller or equal than $\sharp S$ must have projections in special position.

Definition 4.9 A zero-dimensional scheme $Z \subset Y$ is said to be curvilinear if each connected component of $Z$ has embedding dimension 1, i.e., there exists a smooth curve in $Y$ containing $Z$.

We will indeed prove the result even when $S^{\prime}$ is non-reduced, provided that $S^{\prime}$ is curvilinear.

Notice that, of course, any reduced finite subset of $Y$ is curvilinear.

Theorem 4.10 Set $m^{\prime}:=\min \left\{n_{1}, \ldots, n_{k}\right\}$. Fix integers $s>0,0<x<k$ such that $\left(m^{\prime}+1\right)^{k-x} \geq r$. Let $B \subset Y$ be zero-dimensional curvilinear scheme and $S \subset Y$ be a finite 
set with different coordinates such that $\sharp(S)=r, \operatorname{deg}(B)=x$ and $h^{1}\left(\mathcal{I}_{S}^{\mathbf{u}}(1, \ldots, 1)\right)=0$ for any subset $\mathbf{u} \subset\{1, \ldots, k\}$ of cardinality $k-x$.

Assume that each projection $\pi_{i}$ is an isomorphism when restricted to $B$ (when $B$ is reduced this is equivalent to say that also $B$ has different coordinates).

Then, $h^{1}\left(\mathcal{I}_{S \cup B}^{\{1, \ldots, k\}}(1, \ldots, 1)\right)=0$.

Proof Set $Z:=S \cup B$ and assume $h^{1}\left(\mathcal{I}_{Z}^{\{1, \ldots, k\}}(1, \ldots, 1)\right)>0$. Taking $S \backslash(B \cap S)$ instead of $S$ we reduce to the case $S \cap B=\emptyset$. Fix $\mathbf{u}=\{2, \ldots, k\}$ so that $\pi_{\mathbf{u}}$ is the projection to the last $k-1$ factors and write $Y_{\mathbf{u}}=\mathbb{P}^{n_{2}} \times \cdots \times \mathbb{P}^{n_{k}}$

(a) First assume $k=2$ and hence $x=1, \mathbf{u}=\{2\}$ and $\sharp(S) \leq m+1$. Write $B=\{O\}$ with $O=\left(O_{1}, O_{2}\right)$. Take a general hyperplane $H_{1} \subset \mathbb{P}^{n_{1}}$ such that $O_{1} \in H_{1}$ and set $W_{1}:=H_{1} \times \mathbb{P}^{n_{2}}$. Since $H_{1}$ is a general hyperplane containing $O_{1}$ and $S$ has different coordinates, either $W_{1} \cap S=\emptyset$ or $W_{1} \cap S$ is the unique point whose image by $\pi_{1}$ is $O_{1}$. Hence $\sharp\left(W_{1} \cap Z\right) \leq 2$.

Since $\mathcal{O}^{\{1, \ldots, k\}}(1, \ldots, 1)$ is very ample, then $h^{1}\left(\mathcal{I}_{W_{1} \cap Z}^{\{1, \ldots, k\}}(1, \ldots, 1)\right)=0$, so $h^{1}\left(W_{1}, \mathcal{I}_{W_{1} \cap Z, W_{1}}^{\{1, \ldots, k\}}(1, \ldots, 1)\right)=0$. The residual exact sequence of $W_{1}$ in $Y$ gives $h^{1}\left(\mathcal{I}_{Z \backslash\left(Z \cap W_{1}\right)}^{\mathbf{u}}(1, \ldots, 1)\right)>0$. Since $Z \backslash\left(Z \cap W_{1}\right) \subseteq S$, we get $h^{1}\left(\mathcal{I}_{S}^{\mathbf{u}}(1, \ldots, 1)\right)>0$, a contradiction.

(b) Now assume $k \geq 3$ and assume that the claim holds for multiprojective spaces with $k-1$ factors. Write $\{P(1), \ldots, P(y)\}$ for the points of the reduced set $B_{\text {red }}$, where $1 \leq y \leq x$. For any $i \in\{1, \ldots, k\}$ let $\mathcal{A}_{i}$ be the set of all pairs $(P(\mathbf{u}), Q(\mathbf{u})) \in B_{\text {red }} \times S$ such that all the coordinates of $P(\mathbf{u})$ and $Q(\mathbf{u})$ are the same, except the $i$ th one (which is different, because $S \cap B=\emptyset)$. Assume the existence of $(P(\mathbf{u}), Q(\mathbf{v})) \in \mathcal{A}_{i}$ and $(P(\mathbf{u}), Q(\mathbf{v})) \in \mathcal{A}_{j}$. Since $S \cap B=\emptyset, Q(\mathbf{u})$ (resp. $Q(\mathbf{v})$ ) has all coordinates equal to the one of $P(\mathbf{u})$, except the $i$ th (resp. $j$ th) one, which is different. Since $k \geq 3$ and $S$ has different coordinates, we get $Q(\mathbf{u})=Q(\mathbf{v})$. Hence $i=j$. Since $y \leq x<k$, there is $h \in\{1, \ldots, k\}$ with $\mathcal{A}_{h}=\emptyset$. Permuting the factors of $Y$, we may assume $h=1$, i.e., $\mathcal{A}_{1}=\emptyset$. This is equivalent to the injectivity of the map $\pi_{1 \mid S \cup B_{\text {red }}}$. Since $\pi_{1 \mid B}$ is an embedding, we get that $\pi_{1 \mid Z}$ is an embedding.

Fix $P \in B_{\text {red }}$ and call $P_{1}, \ldots, P_{k}$ its components. Take a general hyperplane $H_{1}$ of $\mathbb{P}^{n_{1}}$ containing $P_{1}$. Since $\pi_{1}(B)$ is curvilinear and $H_{1}$ is general, we have $\pi_{1}(B) \cap H_{1}=\left\{P_{1}\right\}$ (scheme-theoretic intersection). Set $W_{1}:=H_{1} \times Y_{\mathbf{u}} . W_{1}$ is an element of $\left|\mathcal{O}_{Y}(1,0, \ldots, 0)\right|$. Since $\pi_{1 \mid B}$ is an embedding and $\pi_{1}(B) \cap H_{1}=\left\{P_{1}\right\}$ (scheme-theoretic intersection), we have $B \cap W_{1}=\{P\}$ (scheme-theoretic intersection). Since $H_{1}$ is general and $S$ has different coordinates, either $W_{1} \cap S=\emptyset$ or $W_{1} \cap S$ is the unique point of $S$ with $P_{1}$ as its first coordinate. Hence $Z_{1}:=Z \cap W_{1}$ is always reduced, it contains $P$ and at most another point, which is in $S$. Set $Z_{\mathbf{u}}:=\operatorname{Res}_{W_{1}}(Z), B_{1}:=\operatorname{Res}_{W_{1}}(B)$ and $S_{1}:=\operatorname{Res}_{W_{1}}(S)=$ $S \backslash\left(S \cap W_{1}\right)$. We have $Z_{\mathbf{u}}=B_{1} \cup S_{1}, \operatorname{deg}\left(B_{1}\right)=x-1, B_{1} \subset B, S_{1} \subseteq S$. Since $\sharp\left(Z_{1}\right) \leq 2$ and $\mathcal{O}^{\{1, \ldots, k\}}(1, \ldots, 1)$ is very ample, we have $h^{1}\left(\mathcal{I}_{Z_{1}}^{\{1, \ldots, k\}}(1, \ldots, 1)\right)=0$. Hence $h^{1}\left(W_{1}, \mathcal{I}_{Z_{1}}^{\{1, \ldots, k\}}(1, \ldots, 1)\right)=0$. The residual exact sequence of $W_{1}$ in $X$ gives $h^{1}\left(\mathcal{I}_{Z_{\mathbf{u}}}^{\{1, \ldots, k\}}(1, \ldots, 1)\right)>0$. Since $\pi_{1 \mid Z}$, is an embedding, then $\pi_{\mathbf{u} \mid Z_{\mathbf{u}}}$ is an embedding. Hence $h^{1}\left(\mathcal{I}_{Z_{\mathbf{u}}}^{\{1, \ldots, k\}}(1, \ldots, 1)\right)=h^{1}\left(Y_{\mathbf{u}}, \mathcal{I}_{Z_{\mathbf{u}}}^{\mathbf{u}}(1, \ldots, 1)\right)$. The inductive assumption on the number of factors of the multiprojective space gives $h^{1}\left(Y_{\mathbf{u}}, \mathcal{I}_{Z_{\mathbf{u}}}^{\mathbf{u}}(1, \ldots, 1)\right)=0$, a contradiction.

Mixing the previous theorem with Proposition 2.4, we get the following. 
Corollary 4.11 Set $m^{\prime}:=\min \left\{n_{1}, \ldots, n_{k}\right\}$ and fix integers $s>0,0<x<k$ such that $\left(m^{\prime}+1\right)^{k-x} \geq r$. Take a tensor $T \in \mathbb{P}^{M}$ with a non-redundant decomposition $S \subset Y$ such that $\sharp S=r$ and $S$ has different coordinates. Assume $h^{1}\left(\mathcal{I}_{S}^{\mathbf{u}}(1, \ldots, 1)\right)=0$ for any subset $\mathbf{u} \subset\{1, \ldots, k\}$ of cardinality $k-x$.

Then any other non-redundant decomposition $S^{\prime} \subset X$ of $T$ of cardinality $\leq x$ cannot have different coordinates.

The statement of Theorem 3.1 cannot produce corollaries on the identifiability, because the assumptions do not include the case $\sharp A=\sharp B$.

We can however modify the proof of Theorem 3.1, adding some assumptions on the decomposition $S$, which produces results which bound different the decompositions of a tensor $T$. Recall that a projective variety $W$ is degenerate if it is contained in some hyperplane.

Definition 4.12 Let $W \subset \mathbb{P}^{r}$ be an integral and non-degenerate projective variety. A finite set (resp. zero-dimensional scheme) $S \subset W$ is said to be set-theoretically quasi-general (resp. scheme-theoretically quasi-general) if the set-theoretic (resp. scheme-theoretic) intersection $W \cap\langle S\rangle$ is set-theoretically (resp. scheme-theoretical) equal to $S$.

Lemma 4.13 Let $W \subset \mathbb{P}^{M}$ be an integral and non-degenerate variety of dimension $n$. Fix a general reduced subset $S \subset W$ with $\sharp S \leq M-n-1$. Then $S$ is the scheme-theoretic base locus of the linear system on $Y$ induced by $H^{0}\left(\mathcal{I}_{S}^{\{1, \ldots, k\}}(1, \ldots, 1)\right)$, i.e $S$ is schemetheoretically quasi-general.

Proof Let $L \subset \mathbb{P}^{M}$ be a general linear space of dimension $M-r-1$. By Bertini's theorem, the scheme $L \cap W$ is a finite set of $\operatorname{deg}(Y)$ points; moreover, the set $L \cap W$ is in linearly general position in $L$. Hence for any $S \subset L \cap W$ with $\sharp S \leq M-r-1$, the restriction of $U:=H^{0}\left(\mathcal{I}_{S}^{\{1, \ldots, k\}}(1, \ldots, 1)\right)$ to $L \cap W$ has $S$ as its set-theoretic base locus. Since $L$ is a linear space, the restriction of $U$ to $W$ has base locus contained in $L \cap W$. Thus $S$ is the base locus of the restriction of $U$ to $W$. Since $W$ is integral and non-degenerate, a general subset $A \subset W$ with cardinality at most $M-n$ spans a general subspace of $\mathbb{P}^{M}$ with dimension $\sharp(A)-1$. The claim follows.

Proposition 4.14 Fix a partition $E \sqcup F=\{1, \ldots, k\}$ with $E=\left\{a_{1}, \ldots, a_{n-h}\right\}$ and $F=$ $\left\{b_{1}, \ldots, b_{h}\right\}$ for some $0<h<k$, and a positive integer $c<M_{F}=\prod_{i=1}^{h}\left(n_{b_{i}}+1\right)$. Let $Z \subset Y$ be a zero-dimensional scheme such that $\sharp(Z)=c, h^{1}\left(\mathcal{I}_{Z}^{E}(1, \ldots, 1)\right)=0$ and $h^{1}\left(\mathcal{I}_{Z}^{F}(1, \ldots, 1)\right)=0$. Assume that $\pi_{F}(Z)$ is set-theoretically (resp. scheme-theoretically) quasi-general. Take any $T \in\langle v(Z)\rangle$ such that $T \notin\left\langle v\left(Z^{\prime}\right)\right\rangle$ for any $Z^{\prime} \subsetneq Z$.

If $S$ is a finite set (resp. zero-dimensional scheme) such that $S \neq Z, \operatorname{deg}(S) \leq b$ and $T \in\langle v(S)\rangle$, then $\operatorname{deg}(S)=b$ and $\pi_{F}(S)=\pi_{F}(Z)$.

Proof By Theorem 3.1, it is sufficient to do the case in which $\operatorname{deg}(S)=c$ and $h^{0}\left(\mathcal{I}_{S}^{F}(1, \ldots, 1)\right)=h^{0}\left(\mathcal{O}_{Y}^{F}(1, \ldots, 1)\right)-b$. In particular $\pi_{F}$ induces an embedding of $S$ into $Y_{F}=\mathbb{P}^{b_{1}} \times \cdots \times \mathbb{P}^{b_{k}}$.

The proof of Theorem 3.1 works verbatim, if there is $H \in\left|\mathcal{O}_{Y}^{F}(1, \ldots, 1)\right|$ containing $S$, but not containing $Z$. Since $h^{0}\left(\mathcal{I}_{S}^{F}(1, \ldots, 1)\right)=h^{0}\left(\mathcal{I}_{Z}^{F}(1, \ldots, 1)\right)$, this is equivalent to require that $H^{0}\left(\mathcal{I}_{S}^{F}(1, \ldots, 1)\right) \neq H^{0}\left(\mathcal{I}_{Z}^{F}(1, \ldots, 1)\right)$, i.e., that $\pi_{F}(S)$ is not contained in the base locus of $\pi_{F}(Z)$. Since $\pi_{F}(Z)$ is the set-theoretic (resp. scheme-theoretic) quasi-general, the base locus of $\left|\mathcal{O}_{Y_{F}}(1, \ldots, 1)\right|$ is $\pi_{F}(Z)$. Thus we get $\pi_{F}(S)=\pi_{F}(Z)$. 
Corollary 4.15 For each $i \in\{1, \ldots, k\}$ fix a set $F_{i} \subset\{1, \ldots, k\}$ such that $i \in F_{i}$ and set $E_{i}:=\{1, \ldots, k\} \backslash F_{i}$. Let $S \subset Y$ be a finite set such that $\sharp(S)=r$, where:

$$
r<\prod_{j \in F_{i}}\left(n_{j}+1\right) \quad \text { and } \quad r \leq \prod_{h \in E_{i}}\left(n_{h}+1\right) .
$$

Take any $T \in\langle v(S)\rangle$, such that $T \notin\left\langle v\left(S^{\prime}\right)\right\rangle$ for any $S^{\prime} \subsetneq S$. Assume that:

$$
h^{0}\left(\mathcal{I}_{S}^{F_{i}}(1, \ldots, 1)\right)=h^{0}\left(\mathcal{O}_{Y}^{F_{i}}(1, \ldots, 1)\right)-r
$$

and

$$
h^{0}\left(\mathcal{I}_{S}^{E_{i}}(1, \ldots, 1)\right)=h^{0}\left(\mathcal{O}_{Y}^{E_{i}}(1, \ldots, 1)\right)-r
$$

for all $i=\{1, \ldots, k\}$. Assume moreover that each $\pi_{F_{i}}(S)$ is set-theoretically (resp. schemetheoretical) quasi-general.

If $S^{\prime} \neq S$ is a zero-dimensional subscheme of degree $\leq r$ such that $\left\langle v\left(S^{\prime}\right)\right\rangle$ contains $T$, then $S^{\prime}$ is a finite set, $\sharp\left(S^{\prime}\right)=r$ and $\pi_{i}\left(S^{\prime}\right)=\pi_{i}(S)$ for all $i$.

Notice that (unfortunately) we are not able to conclude that $S=S^{\prime}$ : they can be different even if $\pi_{i}\left(S^{\prime}\right)=\pi_{i}(S)$ for all $i$. Namely the points can differ by a rearrangement of the coordinates.

Proof Since $h^{0}\left(\mathcal{I}_{S}^{F_{i}}(1, \ldots, 1)\right)=h^{0}\left(\mathcal{O}_{Y}^{F_{i}}(1, \ldots, 1)\right)-\sharp(S)$, each $\pi_{F_{i} \mid S}$ is injective. Assume that $S^{\prime}$ exists. By Proposition 4.14 applied to $F_{i}, S^{\prime}$ is a finite set with $\sharp\left(S^{\prime}\right)=r$, $\pi_{F_{i} \mid S^{\prime}}$ injective and $\pi_{F_{i}}\left(S^{\prime}\right)=\pi_{F_{i}}(S)$. Thus $\pi_{i}\left(S^{\prime}\right)=\pi_{i}(S)$ for all $i$.

Remark 4.16 Corollary 4.15 does not provide the identifiability of a tensor $T$ : it simply bounds strictly the locus where different decompositions of the same tensor $T$ could lie. We observe that, on the other hand, the numerical range of application of Corollary 4.15 is wider than the range of Kruskal's criterion of identifiability.

Just to give an example, consider tensors of type $3 \times 3 \times 6$, corresponding (mod scalars) to points in the space $\mathbb{P}^{53}$ which contains the Segre embedding of $\mathbb{P}^{2} \times \mathbb{P}^{2} \times \mathbb{P}^{5}$. Kruskal's criterion for the identifiability applies only when the rank $r$ is bounded by $r \leq(3+3+6-$ 2) $/ 2=5$. Our Corollary 4.15 applies, taking $F_{1}=F_{2}=\{1,2\}, F_{3}=\{3\}$ and checking the geometric assumptions, even for $r=6$.

Acknowledgements Funding was provided by Gnsaga of Indam.

\section{References}

1. Alexander, J., Hirschowitz, A.: Polynomial interpolation in several variables. J. Algebraic Geom. 4, 201222 (1995)

2. Ballico, E., Bernardi, A.: Decomposition of homogeneous polynomials with low rank. Math. Z. 271, $1141-1149$ (2012)

3. Bernardi, A., Gimigliano, A., Idà, M.: Computing symmetric rank for symmetric tensors. J. Symbolic. Comput. 46, 34-55 (2011)

4. Bernardi, A., Ranestad, K.: On the cactus rank of cubics forms. J. Symbolic Comput. 50, 291-297 (2013)

5. Buczyńska, W., Buczyński, J.: Secant varieties to high degree Veronese reembeddings, catalecticant matrices and smoothable Gorenstein schemes. J. Algebraic Geom. 23(1), 63-90 (2014)

6. Buczyński, J., Ginenski, A., Landsberg, J.M.: Determinantal equations for secant varieties and the Eisenbud-Koh-Stillman conjecture. J. London Math. Soc. 88, 1-24 (2013) 
7. Chiantini, L., Ottaviani, G., Vannieuwenhoven, N.: Effective criteria for specific identifiability of tensors and forms. SIAM J. Matrix Anal. Appl. 38, 656-681 (2017)

8. Chiantini L., Sacchi D.: Segre functions in multiprojective spaces and tensor analysis. In: Casnati G., Conte A., Gatto L., Giacardi L., Marchisio M., Verra A. (eds.) From Classical to Modern Algebraic Geometry. Trends in the History of Science, pp. 361-374. Birkhäuser, Cham

9. Comas, G., Seiguer, M.: On the rank of a binary form. Found. Comput. Math. 11, 65-78 (2011)

10. Comon, P., Golub, G., Lim, L.H., Mourrain, B.: Symmetric tensors and symmetric tensor rank. SIAM J. Matrix Anal. Appl. 30, 1254-1279 (2008)

11. Dersken, H.: Kruskal's uniqueness inequality is sharp. Linear Algebra Appl. 438, 708-712 (2013)

12. Friedland, S.: Remarks on the symmetric rank of symmetric tensors. SIAM J. Matrix Anal. Appl. 37, 320-337 (2016)

13. Gelfand, I.M., Kapranov, M.M., Zelevinsky, A.V.: Discriminants, Resultants, and Multidimensional Determinants. Birkäuser, Zurich (1994)

14. Hauenstein, J.D., Oeding, L., Ottaviani, G., Sommese, A.J.: Homotopy techniques for tensor decomposition and perfect identifiability. J. Reine Angew. Math. (2016). https://doi.org/10.1515/crelle-20160067

15. Kruskal, J.B.: Three-way arrays: rank and uniqueness of trilinear decompositions, with applications to arithmetic complexity and statistics. Linear Algebra Appl. 18, 95-138 (1977)

16. Ranestad, K., Schreyer, F.-O.: On the rank of a symmetric form. J. Algebra 346, 340-342 (2011)

17. Shitov, Y.: A counterexample to Comon's conjecture, Preprint: arXiv: 1705.08740

18. Sidiropoulos, N.D., Bro, R.: On the uniqueness of multilinear decomposition of N-way arrays. J. Chemom. 14, 229-239 (2000)

19. Strassen, V.: Vermeidung von divisionen. J. Reine Angew. Math. 264, 184-202 (1973)

20. Zhang, X., Huang, Z.H., Qi, L.: Comon's conjecture, rank decomposition, and symmetric rank decomposition of symmetric tensors. SIAM J. Matrix Anal. Appl. 37, 1719-1728 (2016) 